\title{
About presence of quasi-oscillatory trend in distribution of QSO's on cosmological distance
}

\section{Vargashkin V.Ya.}

Educational-scientific-research institute of information technology (ESRIIT) of States University - EducationScience-Production Complex (SU-ESPC), Oryol-city, Russian Federation;

E-mail: Vargashkin <varg@ostu.ru>;

This work is devoted search and research of a quasi-oscillatory trend on the schedule of distribution of number of quasars on cosmological distance. Results of the analysis of distribution of quasars on absolute luminosity in vicinities of extreme of a trend are resulted. It is shown, that the oscillatory trend is present at various directions on celestial sphere.

Keywords: quasi-stellar objects, red shift, cosmological distance, statistical distributions, oscillatory trend, luminosity, absolute magnitude

DOI: $10.18698 / 2309-7604-2015-1-523-536$

\section{Introduction}

Elements of the analysis of distribution of quasars on red shift contain (figure 1), in particular, in the tenth edition of the Sloan Digital Sky Survey quasar catalog [1]. The catalogue contains 166.583 quasi-stellar objects with red shift from a diapason from 0.053 to 5.855.
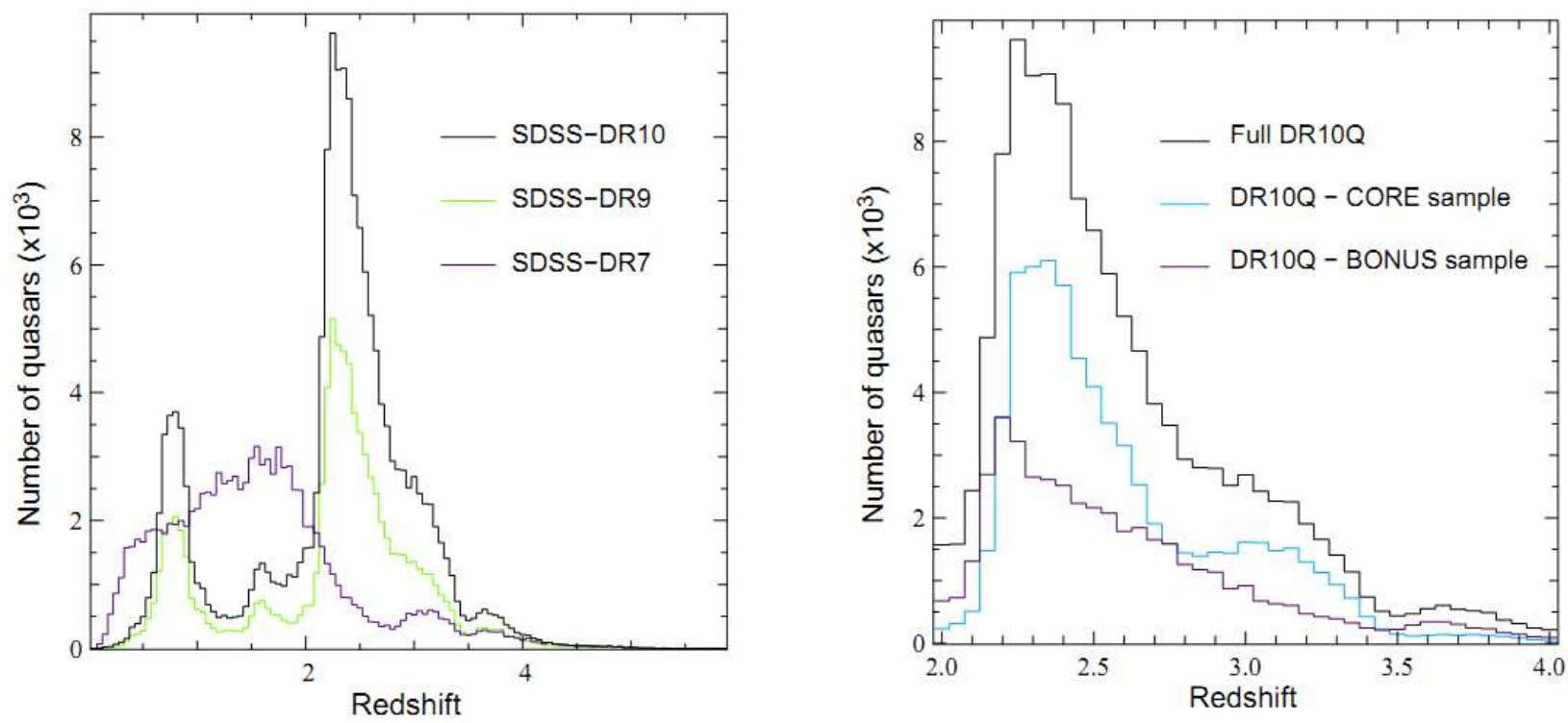

Fig. 1. Distributions of quasars on red shift it agree to data of catalogues [1 - 3]

On the left panel of figure distributions of quasars according to the tenth [1], the ninth [2] and the seventh [3] editions of the catalogue are presented. On the right panel of drawing 
distribution of all set of quasars of the tenth edition, and also the basic and additional parts of this edition are represented. Authors [1] notice, that the distributions represented on the left panel, have two peaks near to values of red shift $z \approx 0,8$, and also $z \approx 1,6$ which are caused by features of measurements.

Therefore for the comparative analysis of repeatability of distributions the data of the catalogue characterized by values $z$ which surpassed 2,0 (the right panel) have been selected. In this area satisfactory similarity of the distributions constructed under catalogues of various releases is observed, and also are constructed on samples of various volume of the tenth edition.

It is necessary to notice, that in allocated with authors on the right panel of figure of area of values $z$ within 2,0 .. 4,0 schedule of distribution of number of quasars looks like, differing from monotonously falling line. On this distribution the quasi-oscillatory component at which local minima close $z \approx 2,8$ are observed, and also $z \approx 3,5$, and maxima close $z \approx 3,1$, and also $z \approx 3,7$ is imposed.

It is necessary to notice also, that the quasi-stellar objects placed in the catalogue are various types of celestial bodies. Quasars, active galactic kernels, and also some classes of stars, in particular, concern them actually. As quasars, as a rule, consider starlike objects, or objects with the starlike kernel, possessing wide issue lines and characterized by the absolute luminosity exceeding on absolute star size value $M_{B}=-22,25$.

Other big types of the quasi-stellar objects which have been not included in the catalogue [1], active galactic nuclei are. To them, in particular, carry Seyfert's galaxies of types $1 \mathrm{~s}$ and $2 \mathrm{~s}$. Active galactic kernels are considered possessing as absolute star size, smaller threshold value $M_{B}=-22,25$.

The third wide type of quasi-stellar objects is $B L$-lacertidae. These objects are characterized by considerable fluctuations of luminosity.

The fourth wide type contains the quasi-stellar objects possessing spectra and luminosity, characteristic for quasars or active galactic kernels, and earlier concerning these classes. Subsequently they have been identified with stars or normal galaxies. These objects are of interest in respect of formation of the fullest set of the remote objects characterized limiting from nowadays revealed values of red shift, reaching several units.

Objects of the second, third and fourth types are not included in the catalogue [1]. 


\section{Quasi-oscillatory trend in distribution of quasi-stellar objects on cosmological distance}

Let's address to data of the catalogue [4]. This catalogue contains data on all to four types of the quasi-stellar objects listed above. In figure 2 distributions on red mixture actually quasars (a), active galactic nuclei (b), $B L$-lacertidae (c), and also the rejected objects (d) are represented. Unlike distributions of figure 1 (the left panel), the presented distributions do not contain features in vicinities of red shift $z \approx 0,8$, and also $z \approx 1,6$. It allows constructing the general distribution of quasi-stellar objects, both on red shift, and on cosmological distance $D$. As in various catalogues for calculation of cosmological distance various values of a constant of Hubble are used, for construction of the subsequent schedules the system, in which $H=1$ and $c=1$ is used.

Distribution of quasi-stellar objects on red shift is shown in figure 3 (a) in the form of the diagram from columns. The distribution histogram is represented in figure 3 (b). The histogram contains the approximating curves, allowing revealing presence on the histogram of systems of maxima and minima. In figure 3 (a) monotonously falling approximating of curves and also six extreme approximating curves which represent a quasi-oscillatory trend of distribution are presented. This trend contains system from three maxima and three minima consistently replacing each other.
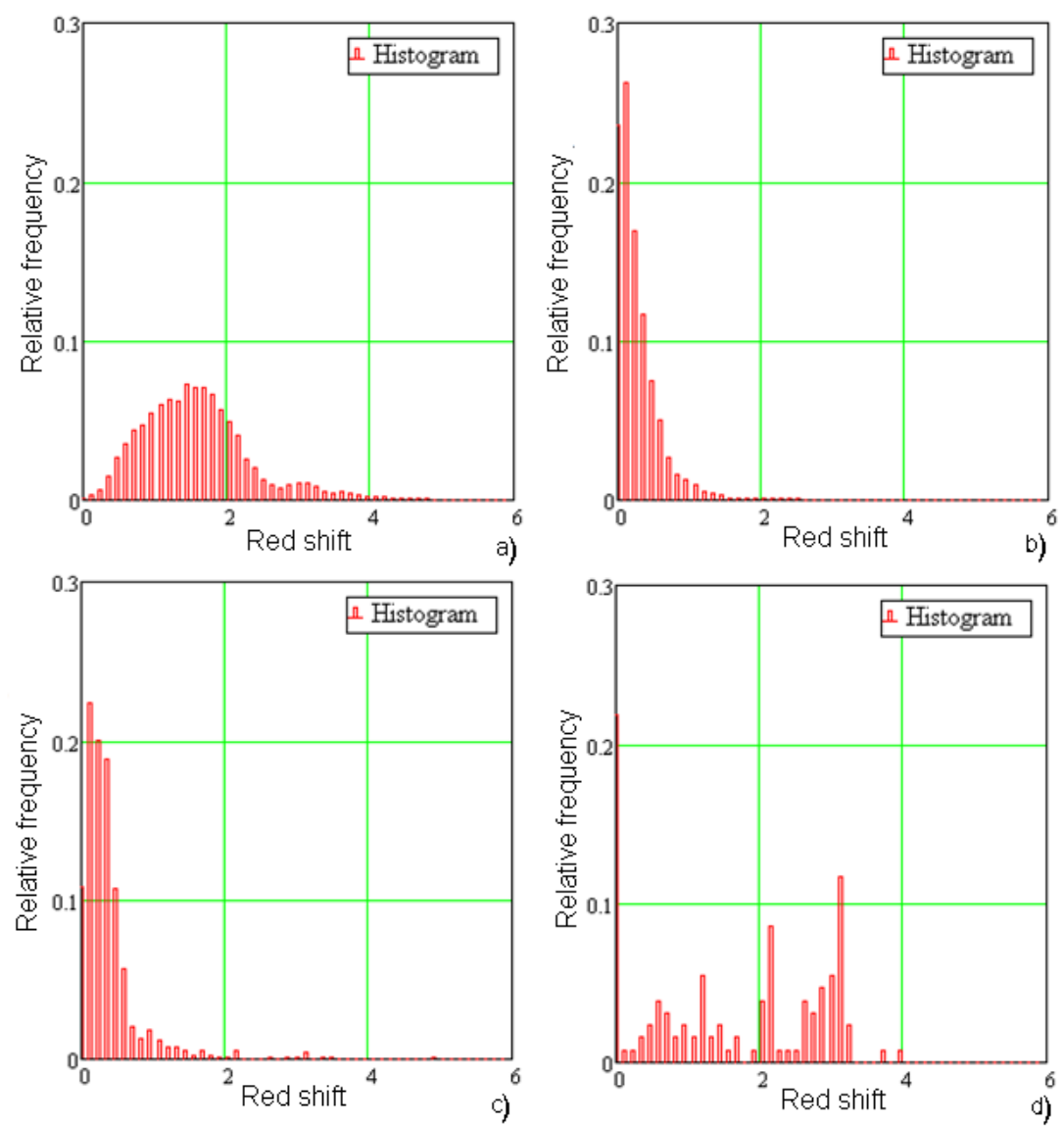
Fig. 2. Distributions on red shift of quasars (a), active galactic nuclei (b), BL-lacertidae (c), and also the rejected objects $(\mathrm{d})$

To state an estimation of capacity of sample for release of 2013 of the catalogue [4], distributions of quasi-stellar objects on cosmological distance for releases of the same catalogue, concerning accordingly to 2006 [5], 2003 [6], 2001 [7], and also have been investigated to 2000 [8]. The statistics of the quasi-stellar objects containing in these catalogues is presented in table 1. Distributions of quasi-stellar objects on cosmological distance for the listed releases of the catalogue with 2000 for 2006 similar to figure3 (a) (2013, [4]), are presented in figure 4.

The analysis of distributions shows, that with increase in number of quasars of their distribution become relatives each other, since figure 4 (b), i.e. about 11 releases [6] at the sample containing more of 60.000 quasars. At volume of the databases, exceeding 100.000 quasars, i.e., for 12 [4] and 13 [5] releases on schedules of distributions the quasi-oscillatory trend is allocated. Scope of the revealed fluctuations reaches $25 \%$ on the relation to background value of monotonous decrease of a curve of distribution. 

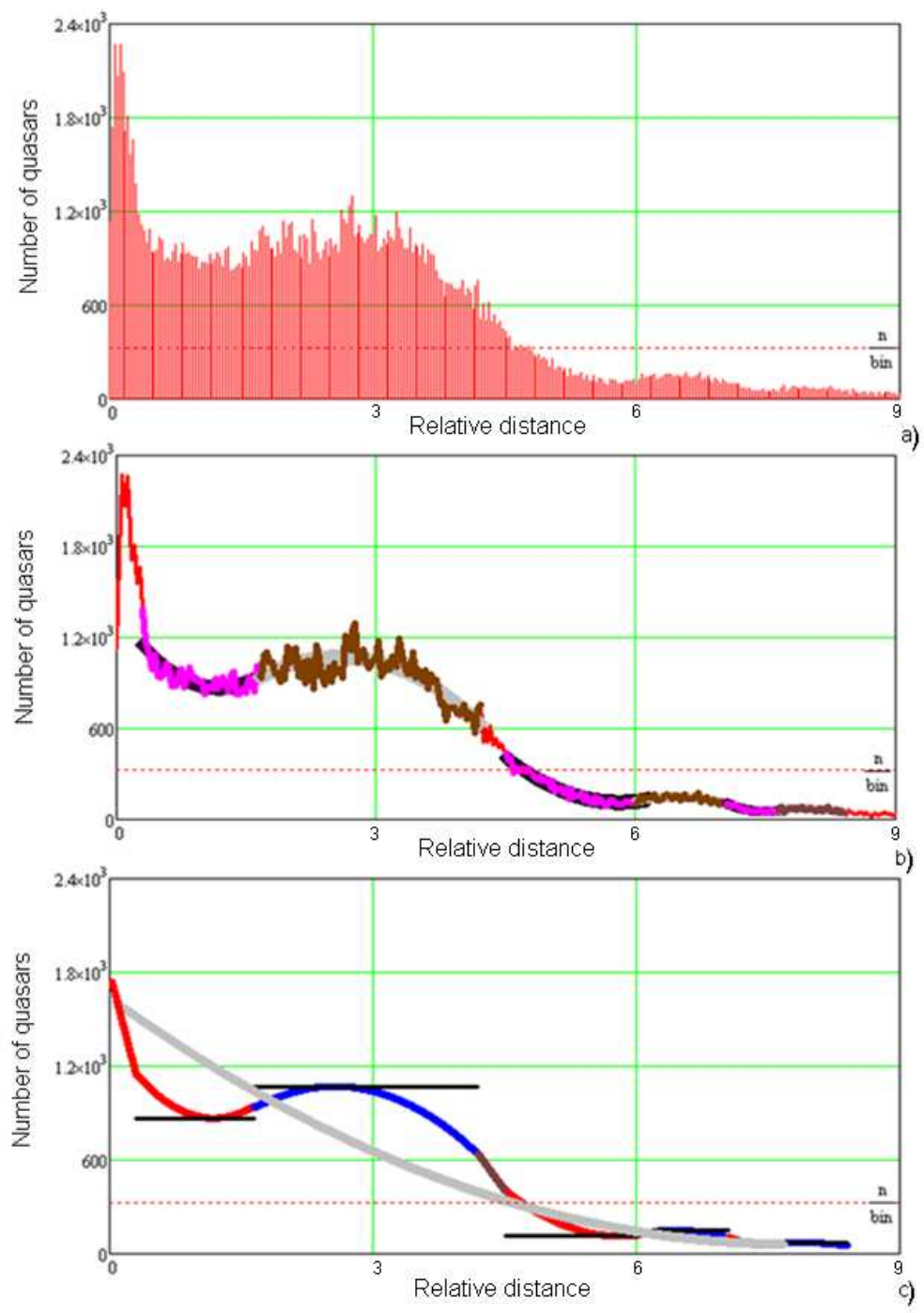

Fig. 3. Distribution of quasi-stellar objects on cosmologic distance $D$, (a), approximation of distribution monotonous and quasi-oscillatory functions (b), and also features of bending around lines (c) 

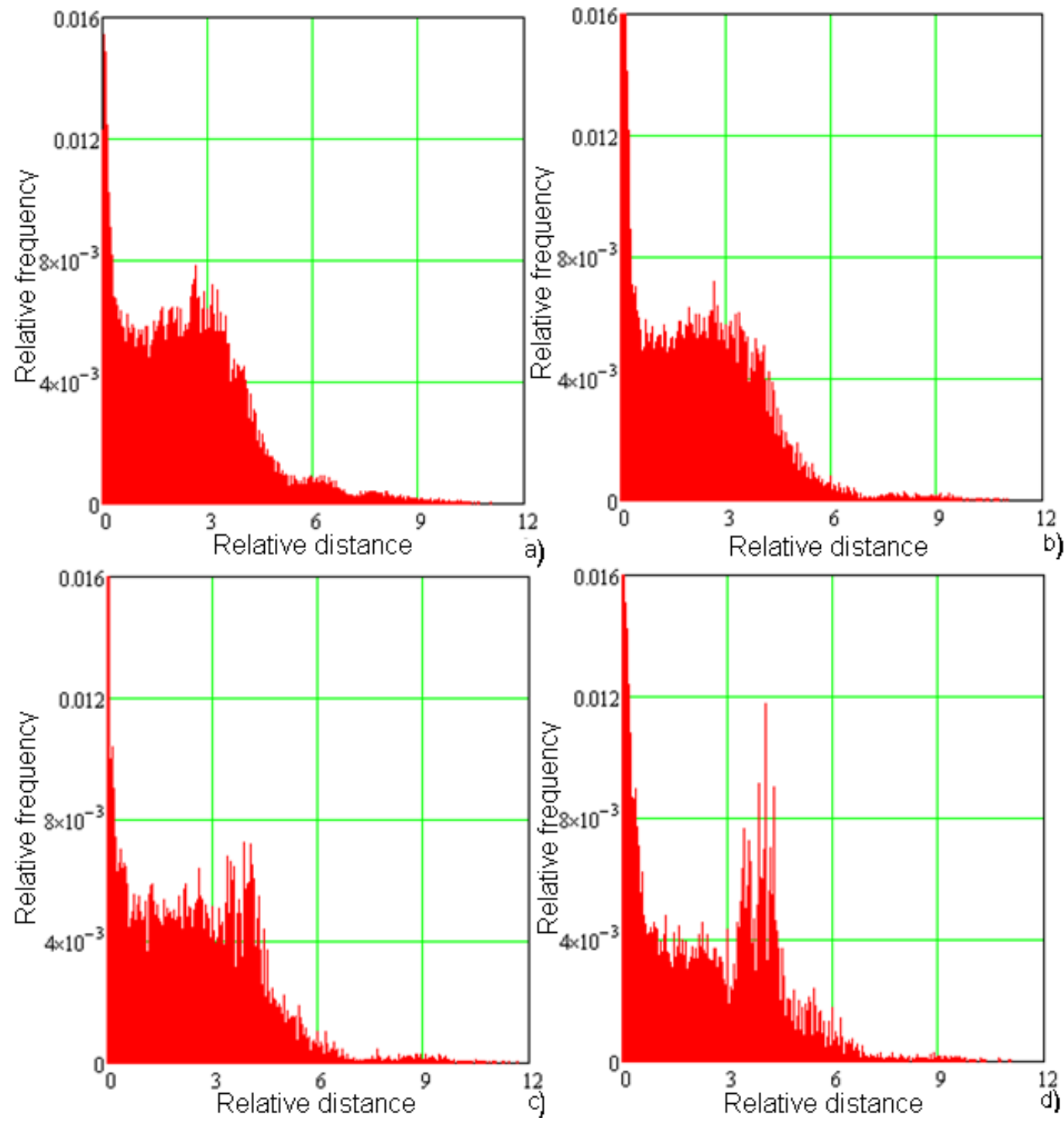

Fig. 4. Distribution of quasi-stellar objects on cosmological distance $D$ for releases 2006 [5], 2003 [6], 2001 [7], 2000 [8] of catalogues [4]

Table 1.-Statistic an of quasi-stellar objects on various releases of the catalogue «Véron-Cetty, M.P. and Véron, P. A Catalogue of Quasars and Active Nucley»

\begin{tabular}{|c|c|c|c|c|c|c|c|}
\hline $\begin{array}{l}\text { Quasars, } \\
\text { number }\end{array}$ & $\begin{array}{l}\text { Active } \\
\text { galactic } \\
\text { nuclei, } \\
\text { number }\end{array}$ & $\begin{array}{l}\text { BL- } \\
\text { lacertidae, } \\
\text { number }\end{array}$ & $\begin{array}{l}\text { Rejected } \\
\text { objects, } \\
\text { number }\end{array}$ & $\begin{array}{l}\text { Total, } \\
\text { number }\end{array}$ & $\begin{array}{l}\text { Distribution, } \\
\text { figure }\end{array}$ & Year & Reference \\
\hline 1 & 2 & 3 & 4 & 5 & 6 & 7 & 8 \\
\hline
\end{tabular}


Proceedings of International Conference PIRT-2015

\begin{tabular}{|l|l|l|l|l|l|l|l|}
\hline 133.336 & 34.231 & 1.374 & 178 & 169.119 & $3(\mathrm{a})$ & 2013 & {$[4]$} \\
\hline 85.221 & 21.737 & 1.122 & 141 & 108221 & $4(\mathrm{a})$ & 2006 & {$[5]$} \\
\hline 48.921 & 15.069 & 876 & 76 & 64942 & $4(\mathrm{~b})$ & 2003 & {$[6]$} \\
\hline 23.760 & 5.751 & 608 & 70 & 30189 & $4(\mathrm{c})$ & 2001 & {$[7]$} \\
\hline 13.214 & 4.428 & 462 & 55 & 18.159 & $4(\mathrm{~d})$ & 2000 & {$[8]$} \\
\hline
\end{tabular}



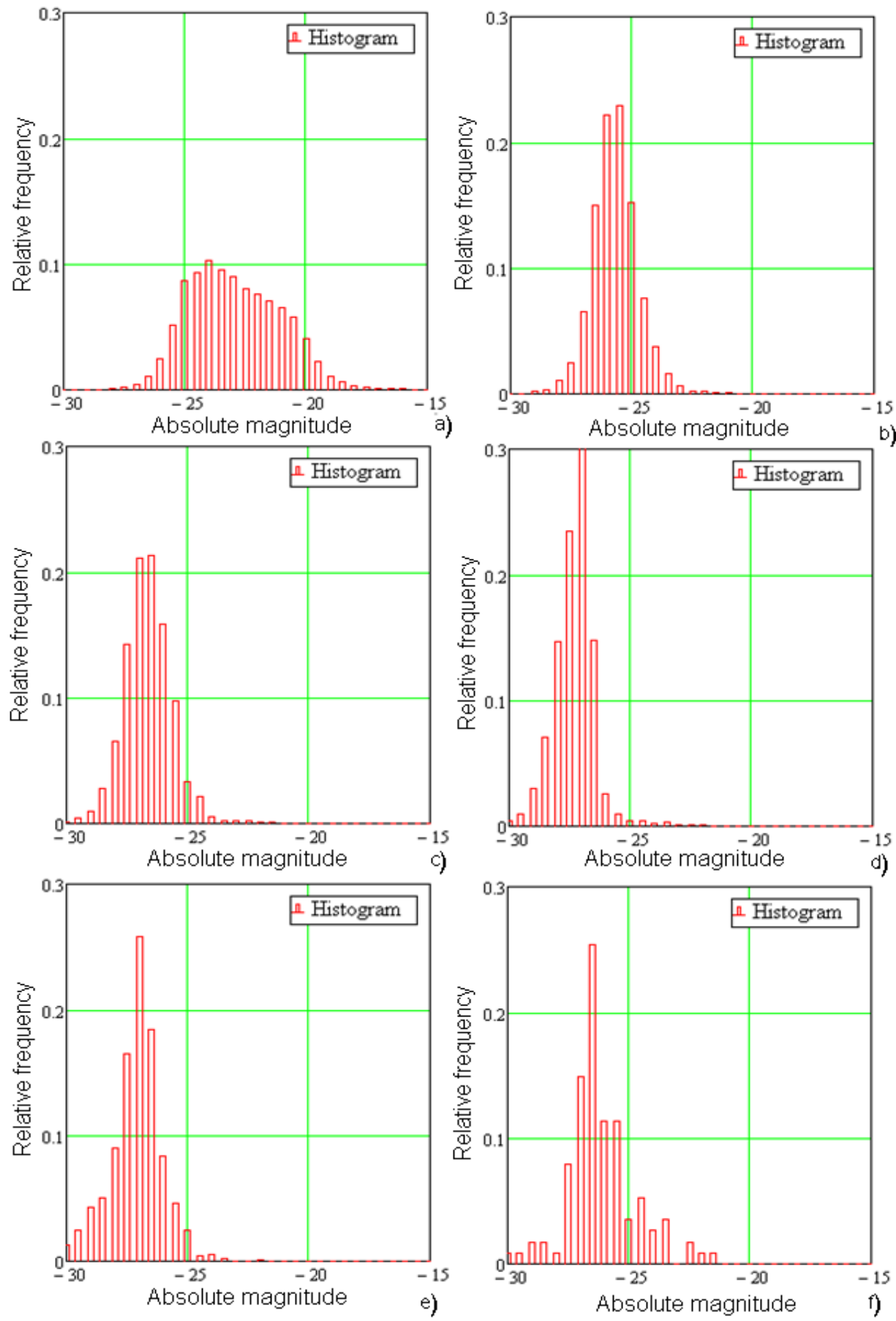

Fig. 5. distribution of quasi-stellar objects on absolute luminosity for $0 \leq \mathrm{Z}<1$ (a); $1 \leq \mathrm{Z}<2$ (b);

$$
2 \leq \mathrm{Z}<3 \text { (c); } 3 \leq \mathrm{z}<4 \text { (d); } 4 \leq \mathrm{z}<5 \text { (e); } 5 \leq \mathrm{z} \text { (f) }
$$

\section{The statistical analysis of a quasi-oscillatory trend}

In figure 5 distributions of quasars on their absolute luminosity for various intervals of red shift are presented. Values of red shift between intervals differ on unit. For red shift at which 
extreme of a quasi-oscillatory trend are observed, with growth of red shift of a maximum of density of distribution of number of quasars towards the big absolute luminosity, i.e. towards smaller values of absolute star size is realized. It is caused by that with growth of red shift $z$ cosmological distance $D$ and consequently, the number of experimentally revealed quasars with rather small absolute luminosity decreases grows. Calculations show, that average quadratic values within adjacent intervals differ slightly.

In figure 6 by three continuous lines character of change of average absolute luminosity of quasars with growth of red shift is shown. The average continuous line corresponds actually to an estimation of average value of absolute luminosity. Two extreme continuous lines, equidistant from central, reflect disorder of absolute luminosity of separate quasars in relation to average value. The distance between average and extreme lines corresponds to one average quadratic deviation of luminosity of quasars.

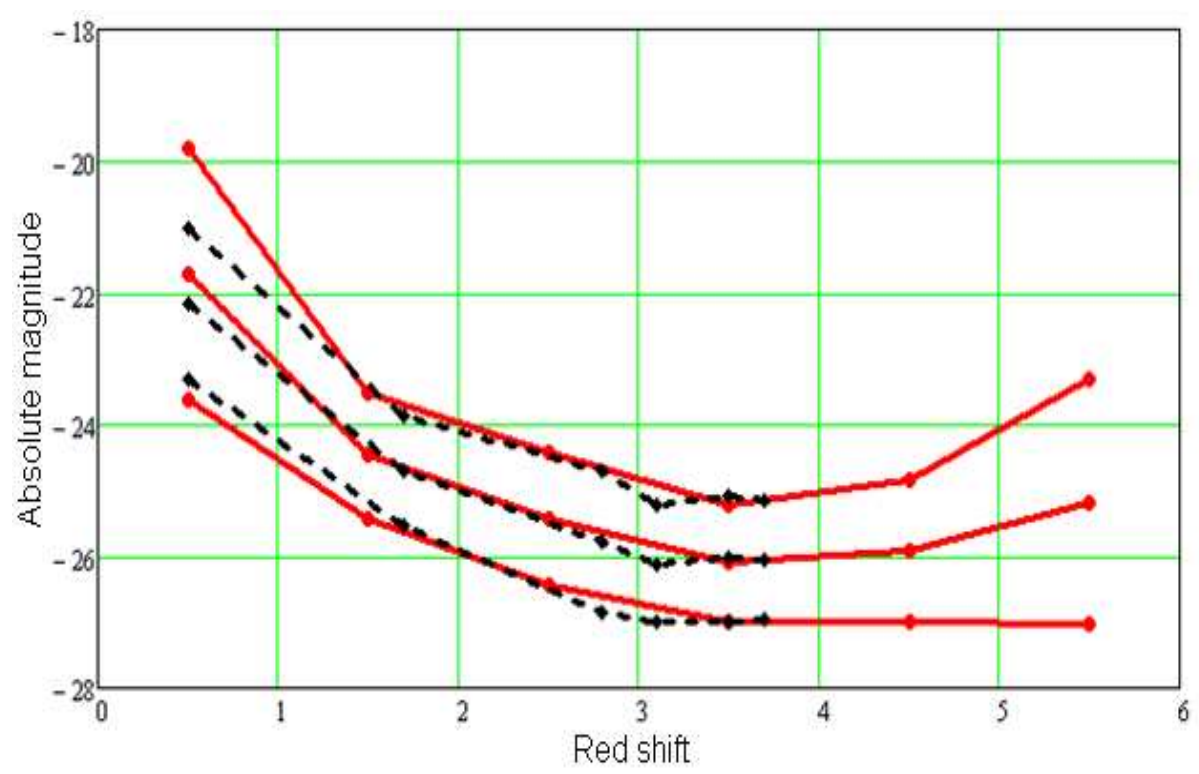

Fig. 6. Character of change of average absolute luminosity of quasars with growth of red shift

It is possible to assume, that statistical properties of absolute luminosity of quasars in vicinities of the red shift corresponding to maxima and minima of distribution of numbers of quasars, represented on figure 3, differ from similar properties at other values of red shift. For check of this assumption from a general totality of absolute luminosity of quasars the partial samples characterizing vicinities of red shift have been taken. The width of vicinities on red shift for first two maxima and minima has been appointed in $\pm 0,1$ symmetrically in relation to red shift of extreme. For the third extreme in view of reduction of number of quasars with growth of red 
shift this width has been appointed in $\pm 0,2$. In figure 7 distributions of quasars on red shift for all six extreme are presented.
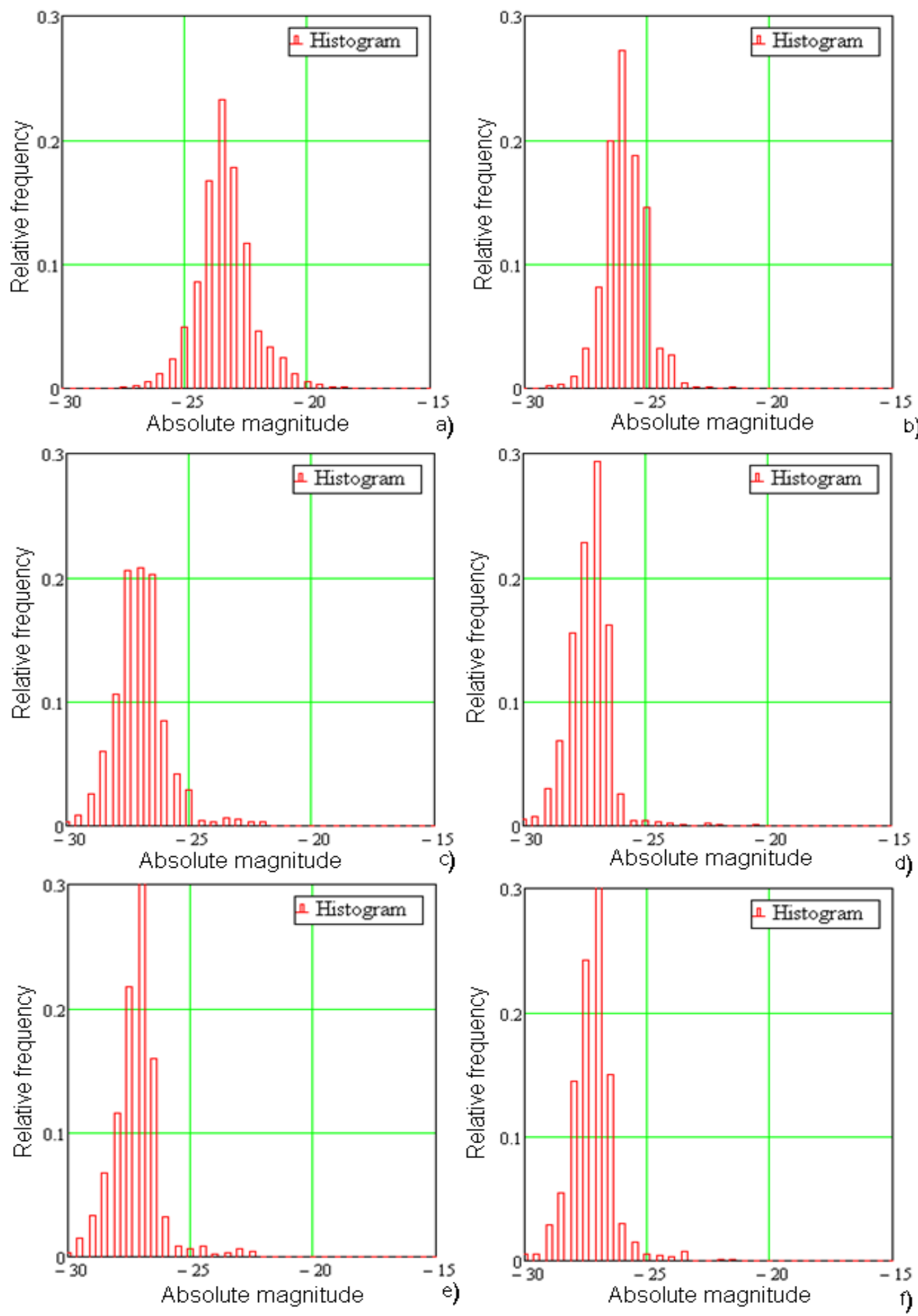

Fig. 7. Distribution of quasars on absolute luminosity for vicinities of the first minimum (a); the first maximum (b); the second minimum (c); the second maximum (d); the third minimum (e); the third maximum (f) 
For these distributions corresponding to maxima and also confidential intervals in one average quadratic deviation from an average has been calculated absolute the luminosity. Results of calculations are put on the schedule of figure 6 in the form of three dashed lines similar on statistical sense to three continuous lines constructed earlier.

The affinity of families' continuous and dashed lines testifies that distributions of quasars on absolute luminosity near to extreme of the schedule of figure 3 are similar to distributions for "background" wide databases on intervals of red shift. Thus, the statistics of luminosity of quasars in areas of space with their big or smaller density rather poorly differs from the similar statistics for other areas of space.

It is necessary to notice, that the revealed quasars are distributed on celestial sphere nonuniformly enough (figure 8).
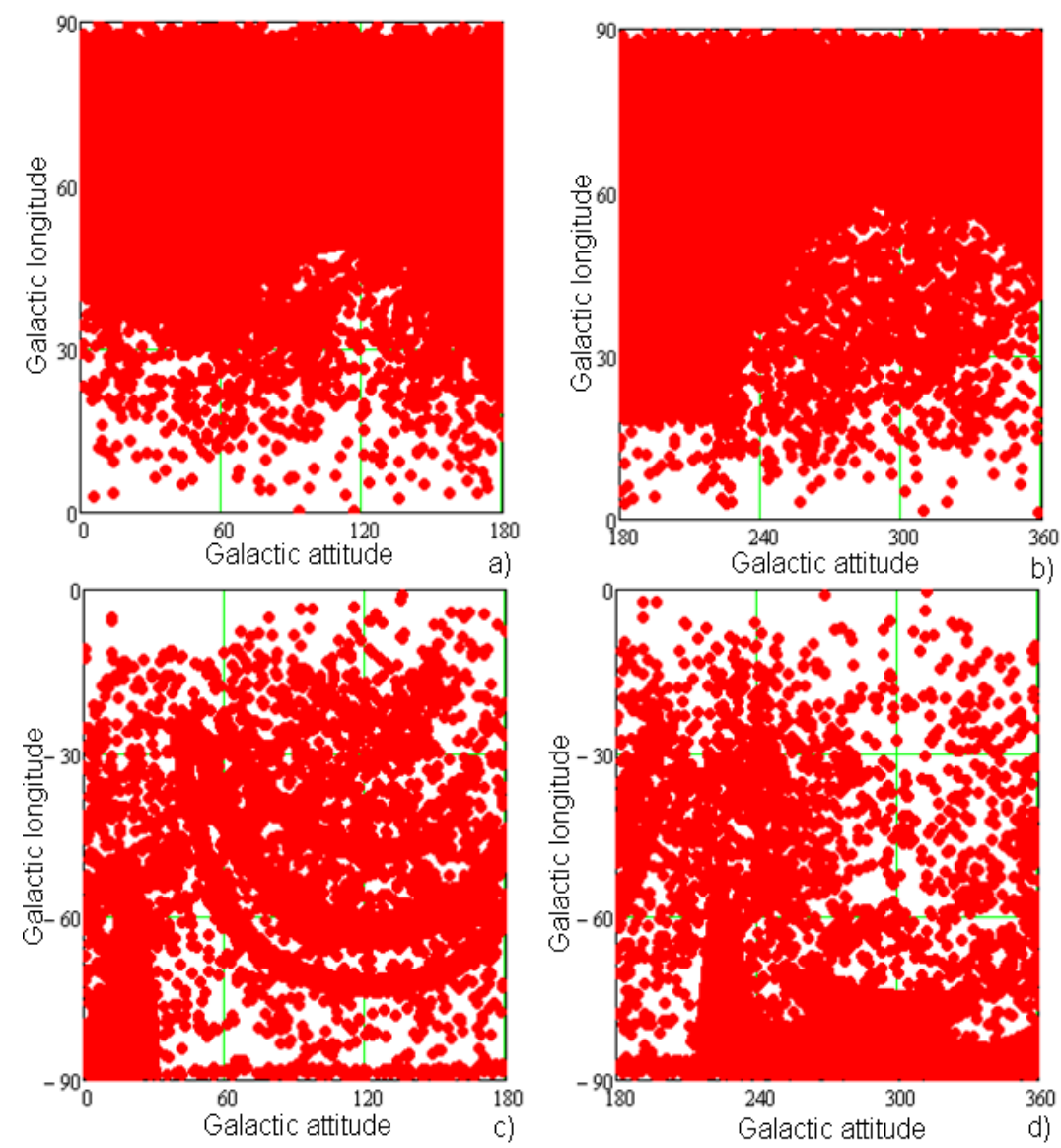

Fig. 8. Distribution of quasars on spherical quadrants of celestial sphere in galactic coordinates 

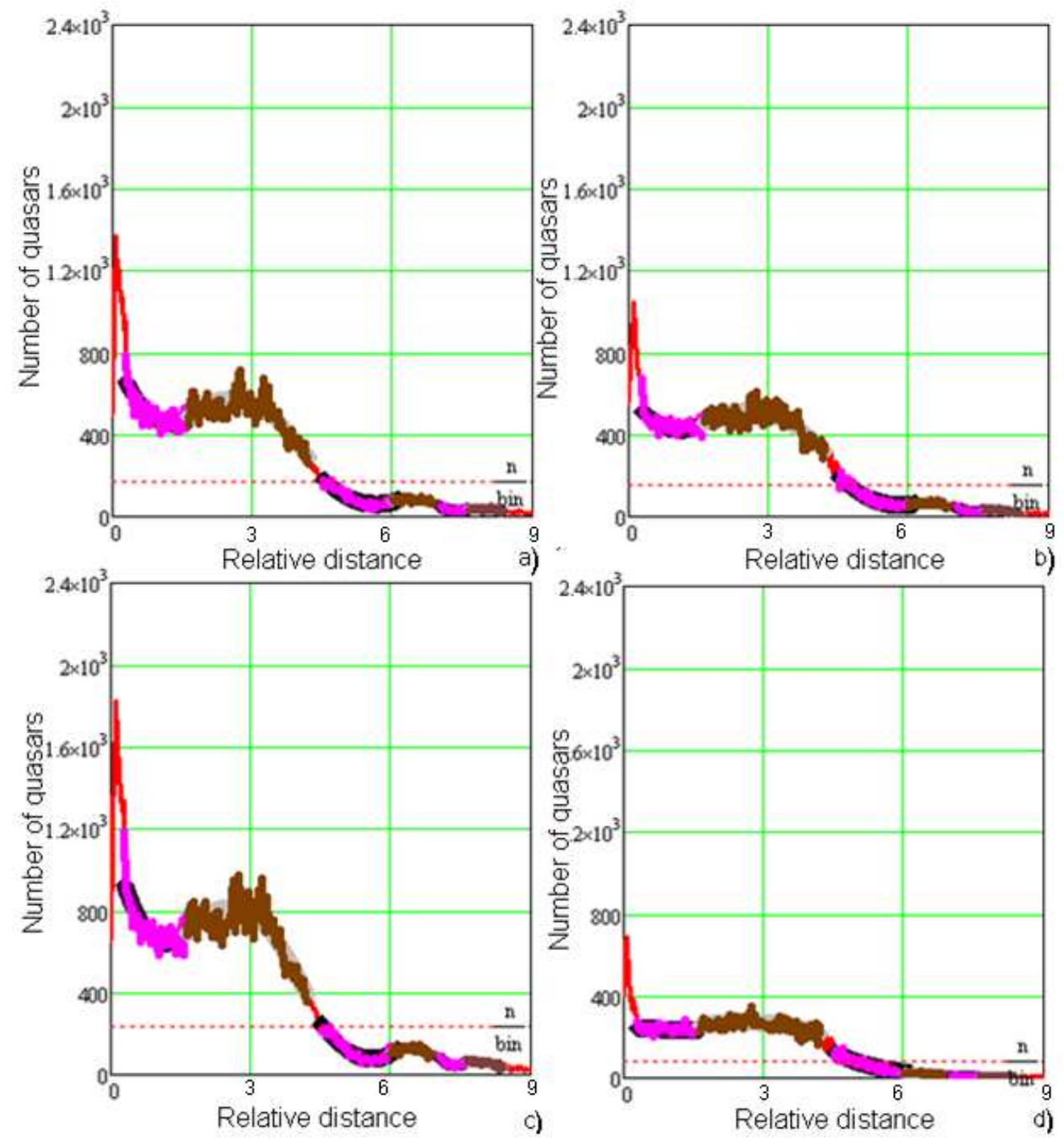

Fig. 9. Distribution of number of quasars on space distance for northern (a), southern (b), western (c) and east (d) hemispheres of celestial sphere agrees figure 8

Table 2. Statistic of an arrangement of abscises of extreme on schedules of figure 3 and 8

\begin{tabular}{|c|c|c|c|c|c|c|c|c|}
\hline $\max 1$ & $\min 1$ & $\max 2$ & $\min 2$ & $\max 3$ & $\operatorname{min3}$ & $\begin{array}{c}\text { total, } \\
\text { number }\end{array}$ & $\begin{array}{c}\text { galactic } \\
\text { attitude }\end{array}$ & $\begin{array}{c}\text { galactic } \\
\text { longitude }\end{array}$ \\
\hline 1 & 2 & 3 & 4 & 5 & 6 & 7 & 8 & 9 \\
\hline 0.0796 & 0.1651 & 0.3683 & 0.4130 & 0.4807 & - & 88203 & $0 \ldots 180$ & $-90 \ldots+90$ \\
\hline 0.0713 & 0.1657 & 0.3798 & 0.4125 & 0.4814 & 0.5051 & 79485 & $180 \ldots 360$ & $-90 \ldots+90$ \\
\hline 0.0764 & 0.1652 & 0.3641 & 0.4156 & 0.4812 & 0.4985 & 124000 & $0 \ldots 360$ & $0 \ldots+90$ \\
\hline
\end{tabular}




\begin{tabular}{|c|c|c|c|c|c|c|c|c|}
\hline 0.0808 & 0.1664 & 0.4364 & 0.3842 & 0.4778 & 0.5066 & 43688 & $0 \ldots 360$ & $-90 \ldots 0$ \\
\hline 0.0770 & 0.1671 & 0.3781 & 0.4197 & 0.4875 & 0.5130 & 167688 & $0 \ldots 360$ & $-90 \ldots+90$ \\
\hline 0.0770 & 0.1659 & 0.3854 & 0.4090 & 0.4817 & 0.5058 & 10061 & \multicolumn{2}{|c|}{ Average value } \\
\hline 4.77 & 0.515 & 7.59 & 3.46 & 0.740 & 1.17 & 46.8 & \multicolumn{2}{|c|}{$\begin{array}{c}\text { normalised average } \\
\text { quadratic deviation, \% }\end{array}$} \\
\hline
\end{tabular}

It is possible to assume also, that presence and position of extreme in figure 3 differ depending on a direction on celestial sphere. In figure 9 schedules of distribution of number of quasars on red shift for northern (a), southern (b), western (c) and east (d) hemispheres of celestial sphere according to figure 8 are represented. Results of the analysis of schedules are placed in table 2 . The table analysis testifies that distribution extreme are present on all schedules of figure 3 and figure 8, and abscises of extreme differ no more than for some percent. Thus, investigated extreme are present at all directions of celestial sphere.

It is necessary to notice also, that the catalogue [4] contains references to 2701 source of various authors. Figure 10 illustrates distribution of sources on values of red shift of the quasars described in these sources. The high density of points of the schedule shows, that the investigated oscillatory trend is present at a database received by numerous authors and consequently, presence of this trend with the big share of probability is not a consequence of a rough error.

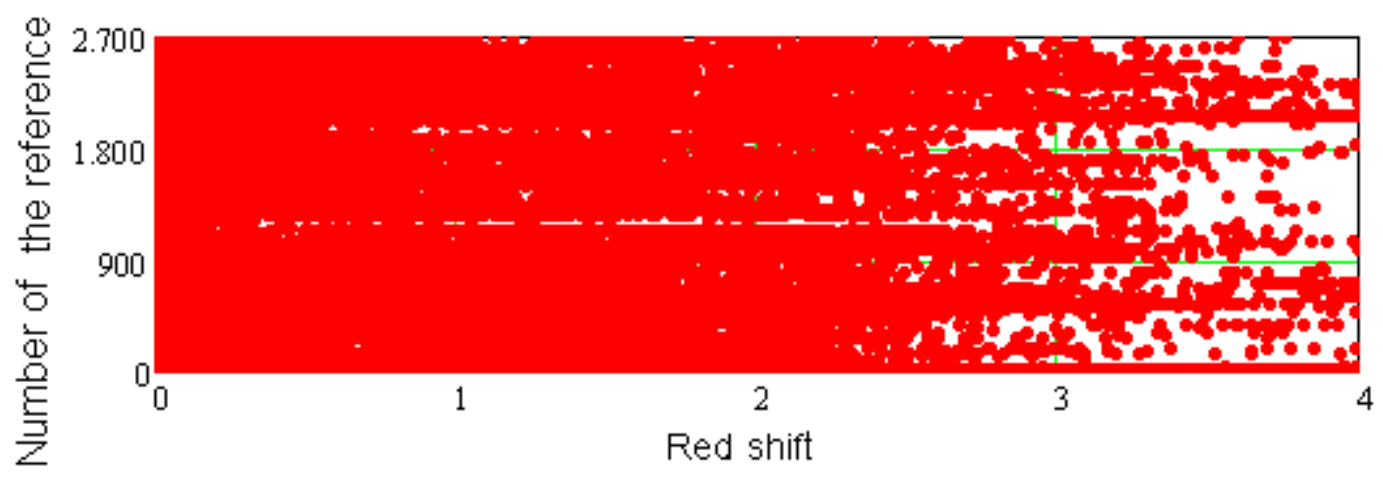

Fig. 10. Distribution of number of sources of the information from the catalogue red shift

\section{Conclusions}

Schedules of distribution of quasi-stellar objects on red shift have a quasi-oscillatory trend in a big way to $20 \%$. This trend within red shift $0 \leq \mathrm{z}<4$ contains three minimum alternating each 
other and a maximum. The analyzed trend is present at samples of quasars of the various catalogues reaching number 50.000 and more. Distribution of quasars on absolute luminosity in vicinities of extreme practically does not differ from similar distributions for adjacent areas of red shift. The revealed alternation of maxima and minima is present also at the samples corresponding to various directions of celestial sphere. Thus abscises of extreme depending on a direction on celestial sphere fluctuate within several percent.

\section{References}

1. Pâris, I., Petitjean, P., Aubourg, É. (2013). The Sloan Digital Sky Survey quasar catalog: tenth data release. $A \& A$, manuscript no. DR10Q, 1.

2. Pâris, I., Petitjean, P., Aubourg, É. (2012). A\&A, 548, A66.

3. Schneider, D.P., Richards, G.T., Hall, P.B. (2010). AJ, 139, 2360.

4. Véron-Cetty, M.-P., Véron, P.A. (2010). Catalogue of Quasars and Active Nucley: 13-th Edition. Astronomy \& Astrophysics manuscript.

5. Véron-Cetty, M.-P., Véron, P.A. (2006). Catalogue of Quasars and Active Nucley: 12-th Edition. ESO Scientific Rcport, YU9.

6. Véron-Cetty, M.-P., Véron, P.A. (2003). Catalogue of Quasars and Active Nucley: 11-th Edition. Astronomy and Astrophysics, 12, 412(2).

7. Veron-Cetty M.-P., Veron P.A. (2000). Catalogue of Quasars and Active Nucley. 9-th Edition. ESO Scientific Report, No. 19.

8. Véron-Cetty, M.-P., Véron, P.A. (1998). A Catalogue of quasars and active nuclei. Edition: $8^{\text {th }}$. ESO Scientific Report Series, Vol. 18. 